\title{
MONITORING AND MANAGEMENT SOFTWARE SYSTEM OF OIL PIPE HYDRAULIC PRESS
}

\author{
Jia Zhu ${ }^{1}$, Anna Ignaczak ${ }^{1}$, Qusai Hassan ${ }^{1}$ \\ ${ }^{1}$ Baicheng Normal University, Baicheng, China
}

\begin{abstract}
In order to solve the problem of balanced tracking control between water pressure and oil pressure in the process of high pressure water test for petroleum pipe, a control system of water pressure test machine is designed on the basis of PLC, industrial control computer and touch screen. The control system has PLC electric control and hydraulic control of electro-hydraulic proportional valve. It has good man-machine visual picture, can dynamically reflect the pressure change, holding time and judgment of qualified steel pipe during the pressing process, and can store the pressing curve, holding time and judgment record of qualified steel pipe, so as to facilitate the search and quality tracking. By introducing the present situation and some structural functions of hydrostatic testing machine, the automatic control scheme of hydrostatic testing machine is put forward, including the design scheme of hydrostatic system, the principle and design scheme of hydraulic control system, and the design scheme of monitoring software. The results show that the visual automatic control system based on VB and STEP7 can satisfy the requirement of the hydrostatic testing machine's pressing process, and realize the real-time monitoring of the field equipment by the upper computer through the free port communication mode. In this way, the advantages of PLC and industrial computer are fully used, which has certain engineering application value. Therefore, the field resources and the existing theoretical knowledge can be effectively used and the design results are in line with the actual situation, which can well meet the market requirements for steel tube inspection equipment.
\end{abstract}

Keywords: Petroleum Steel Pipe, Hydraulic Press, Control System.

\section{Introduction}

In the production of large oilfields, a special pipeline is used.

Now many iron and steel enterprises have invested in the construction of such a production line which is designed specially for processing oil pipelines in oil fields. However, due to the particularity of oil field operation environment, strict requirements are put forward for the performance indicators of special oil pipelines.

At present, many international, regional and national standards organizations in the world have designated their own standards for product production involving different industries.

With the further deepening of reform and opening up, the sustained development of China's economy and the growth of oil and gas demand, and the increasing depth of oil wells, not only the demand for pipelines, fluid pipes, tubing and casing, but also the requirements for steel grade is increasing day by day. At the same time, it also provides a huge market opportunity for oil and gas pipeline manufacturing industry.

Pipeline transportation is the most reasonable and economical mode of transportation of oil and gas. Spiral welded pipe is widely used to transport oil, gas, water and other liquids.

Any minor quality defect of spiral welded pipe will cause great loss in production.

Spiral welded pipe has a very important strategic position and strategic significance in oil and gas transportation.

API is the most authoritative and influential organization in the world's petroleum industry, and its standards have been widely adopted and adopted by ISO all over the world.

An important part of API standard is the traceability of product parameter information [1].

For the hydraulic pressure test system of spiral welded pipe, in order to make it convenient for users to check the parameters information of a pipe when it leaves the factory in the future, it is necessary to save all the technical parameters of the pipe during the hydraulic test.

In this way, it can not only provide users with complete product parameters files, but also urge manufacturers to strictly comply with the standards of production, to ensure product quality.

It is particularly important for hydraulic testing machine to establish a good parameter information preservation and archiving [2]. 


\section{Literature review}

With the continuous improvement of industrial production level, monitoring system has been widely used in hydraulic press, and has achieved remarkable economic benefits. In 2016, Goto et al. used 57-300 PLC and TP270 as the main control equipment to construct a $16 / 22 \mathrm{MN}$ hydraulic press monitoring system, which realized the control and monitoring functions of hydraulic press economically and conveniently [3].

In 2016, according to the production control requirements of $300 \mathrm{MN}$ die forging hydraulic press, Goto et al. also developed a process control system on the basis of WinCC monitoring software. The system realized state monitoring, data acquisition, alarm data recording and processing, and remote setting of important control parameters [4].

In 2017, Chen et al. adopted PC+PLC monitoring system structure to automatically control 100MN multi-direction forging hydraulic press, which ensured the output and quality of products [5]. In 2017, Malygin et al. carried out technical renovation aiming at the problems of long feeding time and low extrusion speed control precision of $12500 \mathrm{~T}$ hydraulic press in a factory. Siemens S7313C-2DP was adopted as the main controller, PLC control system was configured, and PID control algorithm was adopted to realize the accurate control of feeding and extrusion speed.
The communication between OP270 and PLC was realized through DP network, and real-time monitoring of the whole system was carried out [6]. In 2018, Muthuvel et al. adopted BP neural network strategy to control the position of moving beam of hydraulic press. The experimental results show that the parking accuracy has been improved.

From the above research status, it can be seen that the research and application of equipment condition monitoring and control system in China has achieved some results. A monitoring system is built on the basis of PLC. Touch screen and industrial computer are selected as man-machine interface. Since PLC has powerful $1 / 0$ expansion and communication connection ability, configuration software can be used for human-computer interaction to monitor and control the industrial field.

The monitoring system based on industrial computer and programmable controller is becoming more and more popular.

\section{Methodology}

Since hydrostatic testing machine plays an important role in the production process of spiral welded pipe, its control system must meet the needs of spiral welded pipe inspection project.

The structure sketch of the hydrostatic testing machine is shown in the Figure 1.

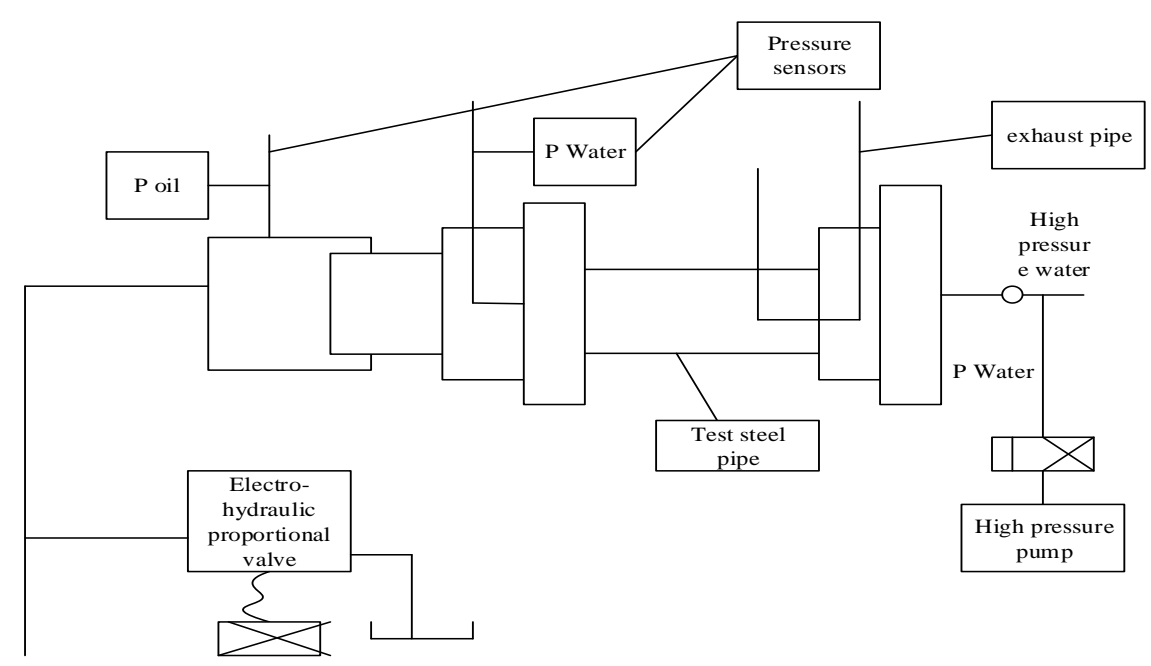

Figure 1: Structure chart of hydraulic press system

In order to make the oil pressure value change with the change of water pressure value, the electrohydraulic proportional valve with follow-up function is adopted. Electro-hydraulic proportional valve is the key component of hydraulic closed-loop control system in hydrostatic testing machine. It transforms the input signal of low power into high-power hydraulic output. The electro-hydraulic proportional valve cooperates with the pressure sensor of feedback detection element to form a closed-loop control system, which can automatically control the output physical quantity with high precision.

The electro-hydraulic control system is mainly composed of proportional amplifier, hydraulic cylinder, pressure sensor, and electro-hydraulic proportional valve. 


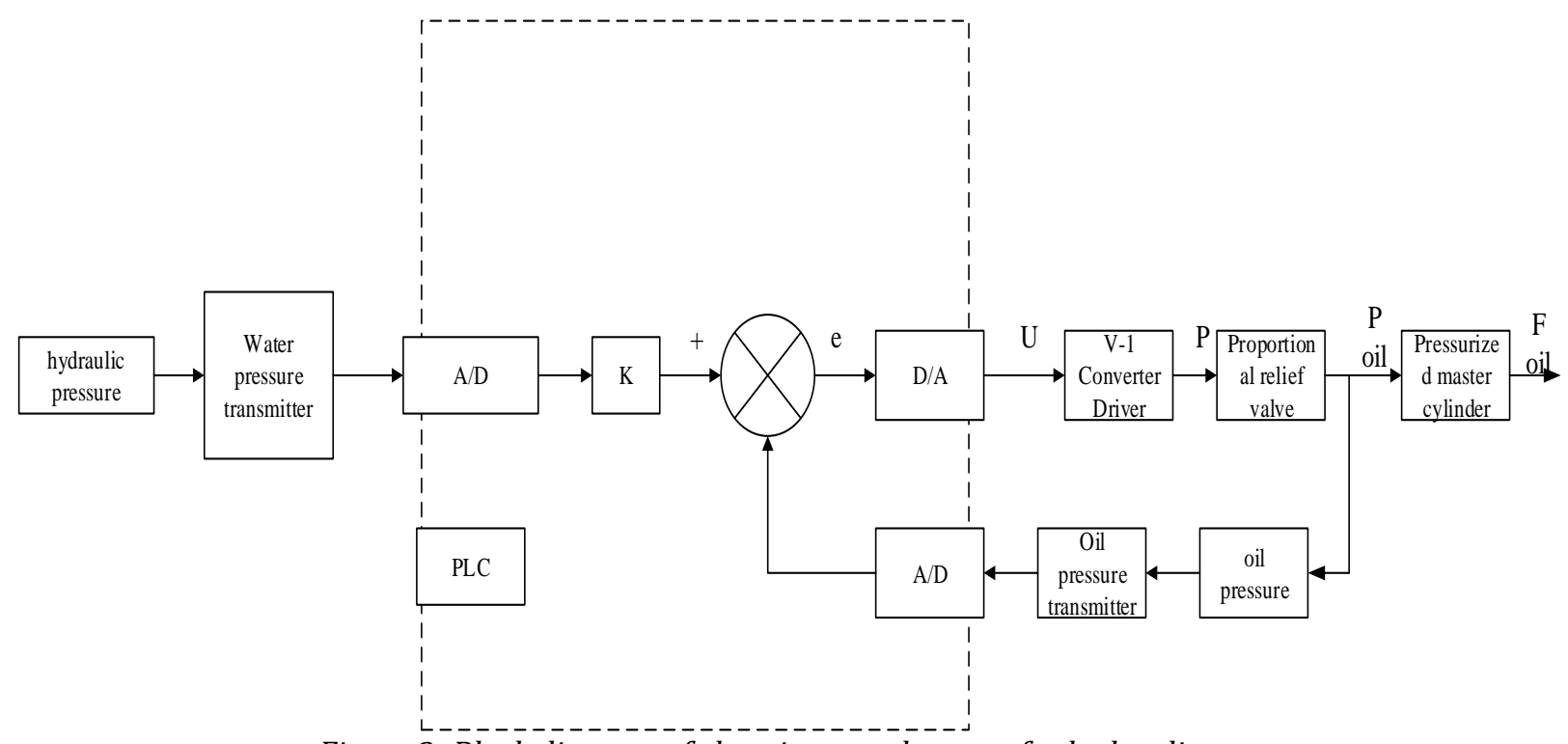

Figure 2: Block diagram of electric control system for hydraulic press

In view of the fluctuation of oil pressure and water pressure in actual operation, it can be concluded that the whole system belongs to a typical follow-up regulation system.

That is, according to the force balance formula:

$$
\mathrm{P}_{\text {oil }} \times \mathrm{S}_{\text {oil }}=\mathrm{P}_{\text {water }} \times \mathrm{S}_{\text {water }}
$$

Among them:

$P_{\text {water: Hydraulic test pressure, Mpa; }}$

Swater: Cross-sectional area of inner diameter of steel tube, $\mathrm{mm}^{2}$;

Poil: In-cylinder pressure of hydraulic testing machine, Mpa;

$S_{\text {oil }}$ : Cross section area of working cylinder of hydraulic testing machine, $\mathrm{mm}^{2}$;

Since the cross-sectional area of inner diameter of steel tube and the cross-sectional area of working cylinder of hydraulic testing machine will not change, there is a coupling relationship between water pressure and oil pressure, which means the change of oil pressure will affect water pressure.

In the actual operation of the system, the fluctuation of water pressure value is large, which makes the oil pressure tracking system change in a larger range, resulting in constant oscillation of water pressure and oil pressure.

During the installation and commissioning of the equipment, the performance curve of the electromagnetic relief valve was accurately calibrated on the spot. Using the calibrated performance curve, the control of test pressure was successfully completed. For the non-linear characteristics, the piecewise linearization method is adopted, and the pressure and current voltage are divided as shown in Table 1.
Table 1: Pressure-current-voltage relation meter

\begin{tabular}{|c|c|c|c|}
\hline $\begin{array}{c}\text { PID } \\
\text { operational } \\
\text { value D/A } \\
\text { (full range } \\
\text { output } \\
32000)\end{array}$ & $\begin{array}{c}\text { Converting } \\
\text { output } \\
\text { voltage (V) } \\
\text { (input of } \\
\text { proportional } \\
\text { valve } \\
\text { drive card) }\end{array}$ & $\begin{array}{c}\text { Proportional } \\
\text { valve input } \\
\text { current (mA) } \\
\text { (proportional } \\
\text { valve drive } \\
\text { card output) }\end{array}$ & $\begin{array}{c}\text { Output } \\
\text { pressure } \\
\text { (MPa) }\end{array}$ \\
\hline 0 & 0 & 0 & 0 \\
\hline 1000 & 0.31 & 80 & 2 \\
\hline 4000 & 1.25 & 140 & 6 \\
\hline 7000 & 2.18 & 200 & 10 \\
\hline 10000 & 3.13 & 260 & 14.5 \\
\hline 14000 & 4.375 & 330 & 21 \\
\hline
\end{tabular}

\section{Realization of Control System Monitoring Software for Hydrostatic Testing Machine}

\subsection{Design of monitoring system}

In order to ensure that the operator can observe all kinds of test data changes in the process of hydraulic test of steel tube from time to time, multiwindow technology is adopted to display on the screen real-time each process of test and pressure, time value of pressure stabilization and pressuretime curve of holding pressure, as shown in Figure 3: 


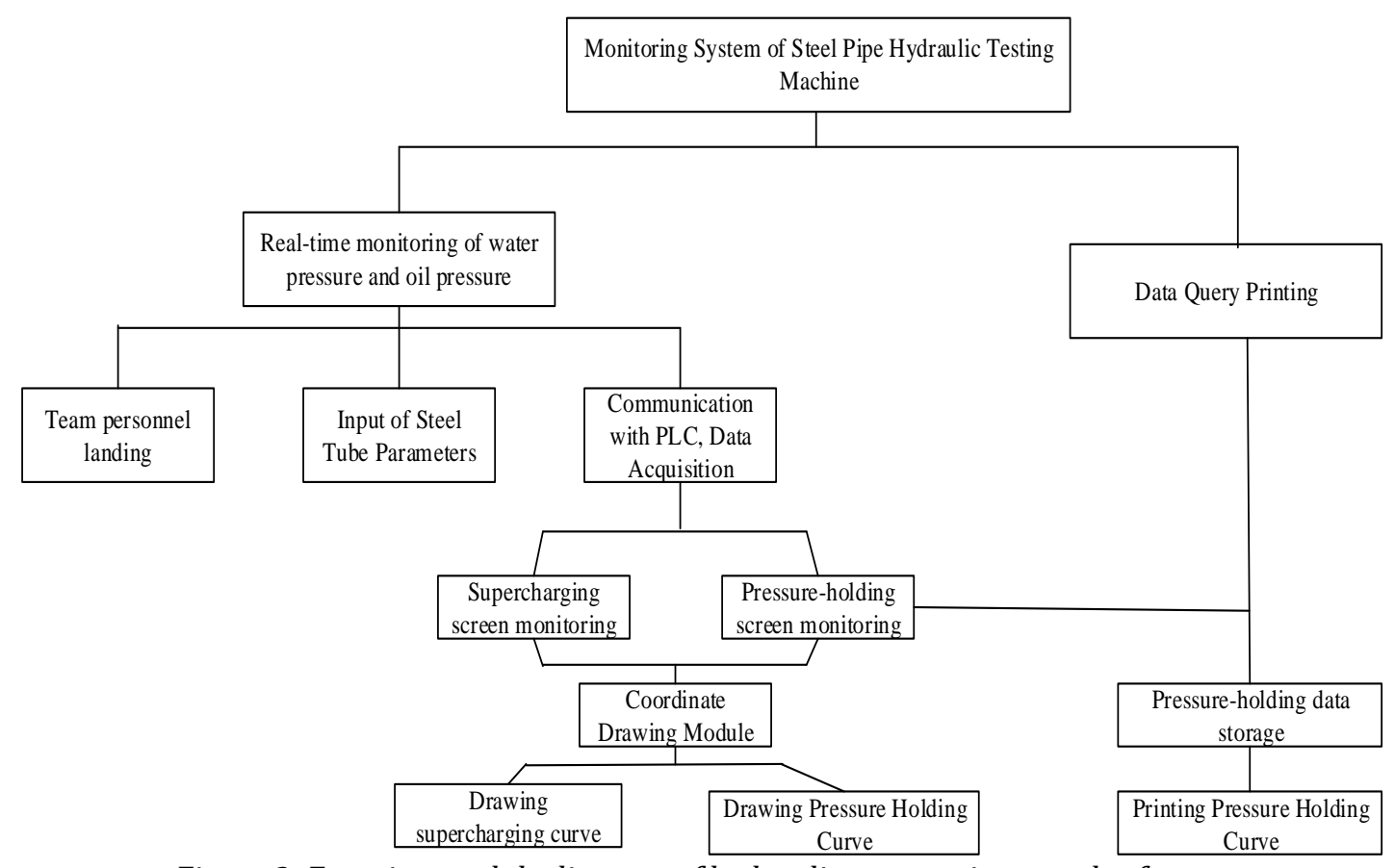

Figure 3: Function module diagram of hydraulic automatic control software

The monitoring system of steel tube hydraulic testing machine can be divided into two parts: one can be used to monitor the hydraulic pressure on site, and the other can be used to look up the historical test data in the office and print them out.

The other also can be used as a field module to query the data just completed at any time.

Because of the multiplicity of query conditions when querying records, the multi-branch judgment technology is used to ensure the accuracy of query when compiling query software. As long as a steel pipe number or specification is input, no matter when the test is done, the hydraulic test data of the steel pipe can be displayed quickly. The data of hydraulic test of steel pipe can be inquired at any time by inputting only the date to be inquired, and can be accurately located in seconds. Inquiry of pressure holding data and curve can be inquired and printed at any time as well as on-site.

The password is set in the database to avoid arbitrary changes.

The data are automatically exported to the database at regular intervals and stored on a fixed hard disk in order to summarize the test situation in time. When printing, the preview function is set up to make full use of the space and print three pressure-holding curves on one sheet of paper. Using database operation programming is not only convenient, but also powerful. Pressure data is stored in Access database, and ADO object is used to create database and data table in VB program. ADO is a new data access method, which not only reduces the access time of a large number of data processing, but also reduces the memory space occupied by data file backup. At the same time, it is very compatible with VB.

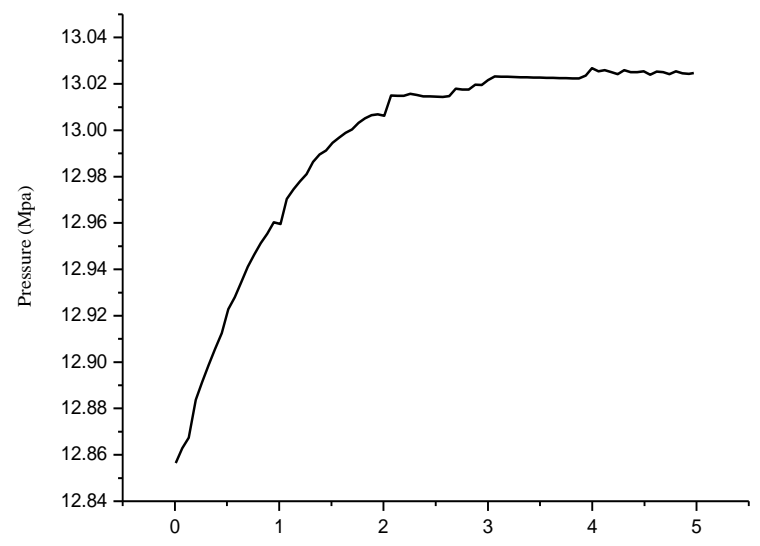

Figure 4: Print chart of pressure holding record

\subsection{Setting up of database}

Background real-time database plays an irreplaceable role in real-time monitoring software.

Whether it is user management or user login interface, historical data analysis, archiving, and report management are all based on this real-time database.

This system establishes the database BYK, MDB furnace number, holding time, prescribed pressure, shift and conclusion in access 2000 time under the conditions of pipe number, material, specification, pressure (1-60), operator. 
Set rstl=Ado Write. Record set

rstl. Add New

rstl. Fields (1) = Format (Date, "yy-mm-dd")

rstl. Fields (2) = Format (Time, "hh:mm:as")

rstl. Fields (3) = SetParameter. Textl (0). Text rstl. Fields (4) = SetParameter. Textl (1). Text rstl. Fields (5) = SetParameter. Textl (2). Text rstl. Fields (6) = SetParameter. Textl (3). Text rstl. Fields (7) = SetParameter. Textl (4). Text rstl. Fields (8) = SetParameter. Textl (S). Text rstl. Fields $(71)=$ logfrm. Worker. Text rstl. Fields (72) = logfrm. work Line. Text

Pipe number, material, specifications, furnace number, holding time and specified pressure have been filled in the parameter setting module. The value of pressure (1-60) is passed in through the control and exists in the database.

The highest pressure, the lowest pressure and the average pressure are obtained by calculating the real-time value and are written into the database. There are three modules.

VB6.0 uses built-in Microsoft Database Jet Engine to access the database. Jet database engine converts the operation on the data access object into physical operation on the database file itself to deal with all kinds of database interface problems. VB6.0 provides three methods of interface with Jet database engine: Data Control, Data Access Object (ADO) and Data Enviroment. ADO model absorbs the essence of two sets of object models of DAO and RDO in VB5.0. Its function is very powerful. Its main advantages are easy to use, high speed and low memory expenditure. It can control the complete programming interface of database. ADO model is used to access and operate database.

\subsection{Programming of communication control module}

The ultimate goal of real-time monitoring software is to monitor the changes of data in the field and to control the switching and analog quantities in the field. If these two things can't be done, the best software interface is just a vase. In addition, the original data in the database of real-time monitoring software is also from the scene.

This actually shows a problem, that is, as a realtime monitoring software, good-looking and intuitive software interface are important, but more important is the real-time communication between the host computer software and the scene, which means the communication problem.

Siemens S7-200 series has excellent performance, high cost performance and wide application range.

Therefore, the communication between Siemens S7-200 series and industrial computer is mainly discussed.
Instructions are written in ASCII code, because an instruction includes not only data, but also necessary control words, including start character, end character, instruction type and so on.

If the data in the instruction is transmitted directly by the binary of its original text, it will inevitably be confused with the control word in the instruction.

For example, the starting character of the instruction is "g", its ASCII code value is $67 \mathrm{H}$, the ending character is "G", and its ASCII code value is $47 \mathrm{H}$.

Table 2: Instruction format of upper computer

\begin{tabular}{|c|c|}
\hline Byte0 & Start character \\
\hline Byte1 & $\begin{array}{c}\text { Instruction type } \\
\text { (read/write) }\end{array}$ \\
\hline Byte2 & $\begin{array}{c}\text { Target PLC station address } \\
\text { (hexadecimal ASCII code) }\end{array}$ \\
\hline Byte3 & \\
\hline Byte12 & $\begin{array}{c}\text { Target register address } \\
\text { (hexadecimal ASCII code) }\end{array}$ \\
\hline Byte13 & Read - \\
Byte29 & $\begin{array}{c}\text { Data to bection } \text { Mritten } \\
\text { (hexadecimal ASCII code) }\end{array}$ \\
\hline $\begin{array}{c}\text { Byte30 } \\
\text { Byte31 }\end{array}$ & $\begin{array}{c}\text { BCC check code } \\
\text { (Hexadecimal ASCII Code) }\end{array}$ \\
\hline Byte32 & End character \\
\hline
\end{tabular}

After receiving the instructions from the host computer, the PLC will send a 21-byte long feedback information. The format is shown in Table 3.

First of all, the starting character marks the beginning of the feedback information. In this case, it is defined as "g" of ASCII code. Different PLC slave stations can define different starting characters, so that the host computer can judge the source of the feedback information according to the starting character of the information.

State information contains state information for instruction execution. In this case, $01 \mathrm{H}$ means correct reading, $02 \mathrm{H}$ means correct writing, $03 \mathrm{H}$ means BCC check code error, and $04 \mathrm{H}$ means illegal instruction. Data area is used to collect the feedback information from B3 to B18. That is the data to be read by reading instruction, which is expressed by hexadecimal ASCII code. BCC check code, which is similar to BCC check code in PC instructions, is the XOR sum of feedback information B3 to B18. The end character, which marks the end of the feedback message, is defined as $26 \mathrm{H}$ in this case. 
Table 3: Feedback information format

\begin{tabular}{|c|c|}
\hline Byte0 & Start character \\
\hline Byte1 & Status information \\
\hline Byte2 & Data area (hexadecimal ASCII \\
Byte3 & \\
Byte4 & \\
Byte5 & \\
Byte6 & \\
Byte7 & \\
Byte8 & \\
Byte9 & \\
Byte10 & \\
Byte11 & \\
Byte12 & \\
Byte13 & \\
Byte14 & \\
Byte15 & \\
Byte16 & \\
Byte17 & ASCII Code) \\
\hline Byte18 & BCC check code (Hexadecimal \\
Byte19 & \\
\hline Byte20 & \\
\hline
\end{tabular}

\subsection{Realization of system communication}

In the aspect of standard serial communication, VB provides a powerful communication control named MSComm1.VBX. The control can set the data transmission and reception of communication, and set the state of serial port and the information format and protocol of serial communication. This control shields the underlying operation in the communication process. Designers can easily realize serial asynchronous communication by setting and monitoring the properties and events of MSComm control. VB can easily develop serial communication program by using ActiveX control MSComm.

MSComm control transmits and receives data through serial port to provide serial communication function for application program. MSCOmm control is very convenient in serial port programming.

Programmers do not need to spend time to understand more complex API functions. They can initialize serial port and input and output data by setting and monitoring the properties and events of MSComm control. MSComm control provides the following two ways to deal with communication. One is event-driven mode and the other is query mode. Event-driven communication is a very effective way to deal with the interaction between serial ports.

These communication events can be captured and processed using OnComm events of MSComm controls. OnComm events can also check and handle communication errors. In the process of programming, you can add your own processing code in OnCo event handler.
The advantage of this method is that the program response is timely and the reliability is high. Each MSComm control corresponds to a serial port. If an application needs to access multiple serial ports, it must use multiple MSComm controls. Query mode is essentially event-driven, but in some cases, it seems more convenient. After each key function of the program, events and errors can be queried by checking the value of the CommEvent attribute. Event-driven and query are integrated to deal with communication.

PLC program module includs the main program module, Read module, Write module, Verify module, Initalize module, RCVcomplete interrupt program, and XMTcomplete interrupt program. Read or Write subroutines are executed when the following three conditions are met. Firstly, the address of the station in the instruction corresponds to the address of the local station. Secondly, the instruction type is read instruction. Thirdly, the BCC check code is correct.

When implementing Read or Write subroutines, RCV must be banned at first. The difference is that the execution of Read subroutines needs to convert the data read by instructions into hexadecimal ASCII codes, write to the sending buffer, calculate BCC check codes, and finally send feedback information.

The execution of Write subroutine directly writes the data in the instruction to the target register, and finally sends the correct feedback information written by the representative.

\section{Conclusion}

On the basis of PLC and industrial control computer, a set of computer monitoring system for hydraulic test tube machine has been designed.

After debugging and field application, it has the following characteristics.

Firstly, the electro-hydraulic proportional control technology used in the application of hydraulic test machine for steel tube has undergone hydraulic test of various specifications, and the control accuracy and sensitivity have been greatly improved.

Secondly, using data processing, PID control algorithm and non-linear correction method, the oil pressure control tracking system can achieve oilwater balance completely and quickly, meet the technical requirements, and better achieve the control of the system.

Thirdly, the hydrostatic pressure test system controlled by computer has a good effect on the pressure curve description, which can better meet the production requirements compared with other methods. 
Fourthly, the free port communication mode not only meets the requirements of real-time rapidity, but also has high communication efficiency. Finally, the computer monitoring system is simple to operate, accurate and reliable, highly automated, economical and practical. In short, the computer control system not only solves the problem of overshoot, but also works stably in the pressureholding section and creates a safe production environment.

Although this hydrostatic testing machine monitoring system is only a relatively simple monitoring system, which cannot be compared with King View, SIEMENS Win CC and in touch, it also involves knowledge of machinery, electronics, control, communication and software, and is a subject worthy of study.

\section{References}

[1] Szurgacz D, "Electrohydraulic control systems for powered roof supports in hazardous conditions of mining tremors", Journal of Sustainable Mining, 2015, Volume 14, Issue 4), Pages 157-163.
[2] Muthuvel P, George B, Ramadass G A, "MagneticCapacitive Wear Debris Sensor Plug for Condition Monitoring of Hydraulic Systems", IEEE Sensors Journal, 2018, Volume 18, Issue 22, Pages 91209127.

[3] Goto K, Itoh T, Nagayama butT, et al, "Development and installation of bedload monitoring systems with submerged load cells", Journal of Mountain Science, 2016, Volume 13, Issue 2, Pages 369-376.

[4] Crotti G, Isidori D, Cigada A, et al, "A hydraulic monitoring system on a bridge over the River Esino, Italy", Journal of Civil Structural Health Monitoring, 2016, Volume 6, Issue 3, Pages 1-8.

[5] Chen W U, Tang Y, Tang L, et al, "Characteristic parameter wireless monitoring system of hydraulic turbine based on Android", Journal of Drainage \& Irrigation Machinery Engineering, 2017, Volume 35, Issue 4, Pages 362-368.

[6] Malygin E N, Karpushkin S V, Glebov A 0, et al, "Vulcanizing Hydraulic Press Heating System for Tempering Metal and Alloy Objects”, Chemical \& Petroleum Engineering, 2017, Volume 53, Issue 5-6, Pages 369-377. 